\title{
Erratum to: Web-Traveler Policies for Images on Social Networks
}

\author{
Anna C. Squicciarini • Smitha Sundareswaran
}

Published online: 17 March 2010

(C) Springer Science+Business Media, LLC 2010

\section{Erratum to: World Wide Web DOI 10.1007/s11280-009-0070-8}

The publisher regrets that the abstract to this article published in Volume 12:4, was incorrect. The corrected version is as follows:

\begin{abstract}
Images are one of the most popular types of contents shared on these sites. Most of these networks offer some rudimentary forms of access controls such as allowing the users to choose who can view their profiles or the images uploaded by them. These controls however apply only in the perimeter of the users' direct control such as desktops, profiles etc. Users have no control over their content once it is downloaded by others. In order to enable a user to truly maintain control over his content, new access control mechanisms must be designed so as to enable users to control their content even when managed by others. Towards fulfilling this gap, in this paper we propose the concept of "web-traveler policies". Web-traveler policies allow the user to specify who can view, upload or download a given image within a social network. The unique, innovative feature of web-traveler policies is that they travel with the image, as long as it is hosted on the given social networking site. Additionally, we explore the possibility of extending these controls across different sites, by using the concept of mashups. In the paper we prove the feasibility of this approach, by implementing a working prototype of our approach on a real open source social network platform. We test the performance and scalability of such architecture under heavy user loads and also its resilience towards naïve attacks. This work is a first innovative step toward scalable systems for providing each user with a centralized system for his/her own content's protection all over the web.
\end{abstract}

The online version of the original article can be found under http://dx.doi.org/10.1007/s11280-009-0070-8.

A. C. Squicciarini $(\bowtie) \cdot$ S. Sundareswaran

College of Information Science and Technology, The Pennsylvania State University, University Park, PA, USA

e-mail: acs20@psu.edu

S. Sundareswaran

e-mail: sus263@psu.edu 